\title{
ARTIFICIAL INTELLIGENCE AND INTELLECTUAL PROPERTY: THE ISSUES OF THE LEGAL REGULATION OF PATENT RELATIONS ${ }^{1}$
}

\author{
Ekaterina V. Kupchina \\ Peoples' Friendship University of Russia, Moscow, Russian Federation
}

\begin{abstract}
Introduction: in the paper, the author analyzed the current problems associated with the use of artificial intelligence in the field of intellectual property. Thanks to the active introduction of this technology in many areas of human activity, there is a rapid growth of innovative processes. On the one hand, such active improvement of the computer technology system creates a favorable environment for the development of economic, political and social relations. On the other hand, however, the potential for the further development of artificial intelligence is of serious concern in the scientific community. In particular, modern digital technologies are developing much faster than the legal framework designed to regulate them is improving. In this regard, the first branch of law that has faced the greatest difficulties is intellectual property law, since it is a branch of law directly related to innovation processes. The purpose of the research is achieved by solving a number of tasks: to determine the role of artificial intelligence as a "subject" of patent relations, as well as the boundaries of responsibility for patent infringement by AI. The methodology is based on a theoretical approach to the study of the legal framework in the field of intellectual property. Based on the analysis of the theoretical data obtained, the author provides the examples of patent violations related to the use of artificial intelligence technology, as well as highlights some modern approaches to solving this problem. The results of the research can be used to determine the key goals and objectives in the law enforcement, research, as well as in educational and teaching activities, in particular, during lectures and seminars on courses in intellectual property law, copyright and patent law. Conclusions: the development of artificial intelligence technologies is central to the development of better intellectual property management systems. The development of new doctrines for new technologies, the modification of the existing patent system, as well as the changes in the policy of intellectual property rights protection contribute to the effective development of innovative processes and the improvement of the legal system as a whole.
\end{abstract}

Key words: intellectual property, artificial intelligence, patents, inventions, property rights.

Citation. Kupchina E.V. Artificial Intelligence and Intellectual Property: The Issues of the Legal Regulation of Patent Relations. Legal Concept = Pravovaya paradigma, 2020, vol. 19, no. 4, pp. 48-54. (in Russian). DOI: https://doi.org/10.15688/lc.jvolsu.2020.4.6

УДК 347.918 .2

Дата поступления статьи: 14.09.2020

ББК 34.047

Дата принятия статьи: 10.10 .2020

\section{ИСКУССТВЕННЫЙ ИНТЕЛЛЕКТ \\ И ИНТЕЛЛЕКТУАЛЬНАЯ СОБСТВЕННОСТЬ: ВОПРОСЫ ПРАВОВОГО РЕГУЛИРОВАНИЯ ПАТЕНТНЫХ ОТНОШЕНИЙ ${ }^{1}$}

\author{
Екатерина Валентиновна Купчина \\ Российский университет дружбы народов, г. Москва, Российская Федерация
}

Введение: в настоящей статье автором были проанализированы текущие проблемы, связанные с при-
денением искусственного интеллекта в сфере интеллектуальной собственности. Благодаря активному вне-
вационных панной технологии во многие сферы деятельности человека происходит стремительный рост инно-
логий создает благоприятную среду для развития экономических, политических и социальных отношений.
Однако, с другой стороны, потенциал дальнейшего развития искусственного интеллекта вызывает серьез- 
ную озабоченность в научной среде. В частности, современные цифровые технологии развиваются значительно быстрее, нежели совершенствуется правовая база, призванная их регулировать. В этой связи первая отрасль права, которая столкнулась с наибольшими трудностями - это право интеллектуальной собственности, так как является отраслью права, непосредственно связанной с инновационными процессами. Цель настоящего исследования достигается путем решения ряда задач: определить роль искусственного интеллекта, как «субъекта» патентных отношений, а также границы ответственности за нарушение патентных прав со стороны AI. Методология основывается на теоретическом подходе к исследованию правовой базы в сфере интеллектуальной собственности. На основе анализа полученных теоретических данных в настоящей работе автором приводятся примеры нарушений патентных прав, связанных с применением технологии искусственного интеллекта, а также освещаются некоторые современные подходы к решению данной проблемы. Результаты исследования могут быть использованы при определении ключевых целей и задач в правоприменительной, научно-исследовательской деятельности, а также в учебно-преподавательской деятельности, в частности, при лекциях и семинарских занятиях по курсам права интеллектуальной собственности, авторского и патентного права. Выводы: развитие технологий искусственного интеллекта является центральным звеном в разработке более совершенных систем управления интеллектуальной собственностью. Разработка новых доктрин для новых технологий, модификация существующей патентной системы, а также изменение политики защиты прав интеллектуальной собственности способствуют эффективному развитию инновационных процессов и совершенствованию правовой системы в целом.

Ключевые слова: интеллектуальная собственность, искусственный интеллект, патенты, изобретения, право собственности.

Цитирование. Купчина Е. В. Искусственный интеллект и интеллектуальная собственность: вопросы правового регулирования патентных отношений // Legal Concept = Правовая парадигма. $-2020 .-$ T. 19, № 4. C. 48-54. -DOI: https://doi.org/10.15688/lc.jvolsu.2020.4.6

\section{Роль искусственного интеллекта в интеллектуальной собственности}

Еще совсем недавно упоминание о технологиях искусственного интеллекта (Artificial Intelligence - AI) можно было встретить лишь на страницах фантастических романов. В настоящее время данная технология представляет собой неотъемлемую часть современного мира, являясь одним из ключевых катализаторов и движущих сил цифровой экономики. Безусловно, роль искусственного интеллекта в современном обществе нельзя недооценивать. Большинство ученых, которые ведут свои исследования в данной области, сходятся во мнении, что AI может превзойти человека во многих сферах деятельности уже в последующее десятилетие [8, с. 4437]. Мало кого сейчас можно удивить беспилотными автомобилями или программами для перевода, основу которых составляют технологии искусственного интеллекта. Уже большинство сфер человеческой жизнедеятельности, таких как здравоохранение, образование, транспорт и некоторые отрасли промышленного производства, неотъемлемо связаны с технологиями AI, существенно упрощая и делая их использование более доступным и эффективным.
До сих пор не существует единого определения искусственного интеллекта. Некоторые специалисты определяют его в широком смысле как компьютеризированную систему, демонстрирующую поведение, которое обычно считается требующим интеллекта, в то время как другие определяют AI как систему, способную принимать рациональные решения сложных проблем или принятия соответствующих действий для достижения своих целей в реальных условиях [11, с. 15].

Несомненно, что технологии искусственного интеллекта тесно и непосредственно связаны с правом интеллектуальной собственности (Intellectual Property - IP), так как это отрасль права, которая призвана регулировать отношения в сфере инноваций и цифровых технологий. На сегодняшний день при помощи глубокого машинного обучения (Deep Learning DL) компьютеры могут создавать колоссальные массивы контента, помогая обрабатывать огромные объемы цифровой информации, например, в сфере предпринимательских отношений и даже судебной системе. Стремительное развитие технологий искусственного интеллекта обусловливает стремительный рост частных и государственных инвестиций в их развитие, а также в разработку новых технологичес- 
ких решений на основе искусственного интеллекта $[6$, с. 3]. С появлением компьютеров наступила эра интеллектуальных машин, которые позволили не только автоматизировать многие процессы, но и привели к возникновению сложных взаимосвязанных систем. Такие системы захватили наш образ жизни и, помогая решать повседневные задачи современного человека, все больше увеличивают зависимость от них [4, c. 32].

Ключевой особенностью искусственного интеллекта является его способность к самообучению. Анализируя данные, полученные на собственном опыте, AI способен решать многие аналитические задачи, такие как: оценка, классификация, прогнозирование и кластеризация данных. Пожалуй, на сегодняшний день DL является наиболее прогрессивной технологией. К первому уровню применения AI относятся наиболее известные в настоящее время:

- маркировка видео;

- машинный перевод.

Ко второму уровню возможностей искусственного интеллекта относится способность к восприятию окружающей среды сродни человеческим сенсорным функциям, таким как зрение, слух и устная речь. В свою очередь, под компьютерным зрением понимается разновидность восприятия объектов, обеспечиваемое алгоритмами DL. Данная технология успешно применяется во многих областях, например, распознавании лиц при поиске преступников или пропавших людей. В сфере здравоохранения компьютерное зрение нашло свое применение при диагностировании заболеваний. Так, например, американские ученые разработали алгоритм, который оценивает рентген грудной клетки на предмет признаков болезни. За весьма короткий срок их разработка превзошла опытных радиологов при диагностике пневмонии [12, с. 15].

В области распознавания речи алгоритмы DL достигли точности более $95 \%$. Большинство современных смартфонов, умных часов и игровых консолей имеют возможность воспринимать и определять речь человека. На сегодняшний день это является наиболее прогрессивной областью компьютерного восприятия с широким спектром вариантов использования, начиная от языкового моделирования и классификации текста до машинного перевода в разговорные системы, так называемые Chatbots [13, c. 670].

По мере перехода от простых компьютерных технологий к искусственному интеллекту возникает вопрос о правовых последствиях такой технологии. Во всем мире сейчас идет «глобальная гонка» за построение таких систем, что порождает множество дискуссий, касающихся государственной политики, которая должна защищать широкие интересы общества. Наиболее обсуждаемыми в данной связи вопросами являются: государственно-частное партнерство, повышение доступности ресурсов, анонимность данных, приложения искусственного интеллекта и т. д. Все эти вопросы обусловливают потребность в сильной государственной поддержке, которая будет способствовать активному развитию искусственного интеллекта и интеллектуальной собственности, а также защищать потребности общества [14, с. 605].

В этой связи встает вопрос о необходимости совершенствования правовой базы интеллектуальной собственности, которая должна обеспечивать эффективное регулирование отношений, возникающих в результате активного внедрения технологий искусственного интеллекта [2, с. 130]. В настоящее время наиболее острым является вопрос правового регулирования патентных отношений, связанных с применением технологий AI.

\section{АI как «субъект» патентных отношений}

Большинство патентных законов создавались в то время, когда компьютеры еще не существовали. Сегодня компьютеры и программное обеспечение стали неотъемлемой частью цифрового общества. Недостаточная подготовка патентного законодательства к грядущим столкновениям с искусственным интеллектом может привести к негативным социальным, экономическим и этическим последствиям. Несмотря на постоянные изменения в патентном законодательстве, эволюция технологий происходит более быстрыми темпами [3, с. 90].

Анализируя современные тенденции по внедрению технологии искусственного интел- 
лекта, можно выделить четыре основных вопроса патентного права, на которые оказывает непосредственное влияние АI:

- соответствие объектам патентования технологий искусственного интеллекта;

- патентоспособность изобретений, созданных с помощью AI;

- ответственность за нарушение патентных прав со стороны AI;

- роль искусственного интеллекта, как «субъекта» патентных отношений.

Вопрос права собственности, то есть кто является законным обладателем прав интеллектуальной собственности на изобретения: создатель алгоритмов или компьютер, обладающий искусственным интеллектом, среди всех стоит наиболее остро. Среди ученых и программистов по всему миру происходят постоянные попытки «легализовать» и признать право авторства за AI. Такое признание может иметь самые непредсказуемые и далеко идущие последствия не только для патентной системы, но и всего права интеллектуальной собственности. В данном ключе некоторые ученые-правоведы заявляют, что необходимо кардинально менять систему, отменяя старые законы и принимая новые, предвещая волну инноваций. Другие сходятся во мнении, что подобные меры могут иметь крайне негативные и беспрецедентные последствия [9, с. 10].

Рассмотрим для примера случай, произошедший в США с системой DABUS. Разработчиком данной системы является эксперт по искусственному интеллекту Стивен Талер. В общем смысле данная система представляет собой искусственную нейронную систему, имитирующую творческий процесс человека. Получая определенную информацию, DABUS генерирует ее в идеи. Системой были созданы два изобретения: первое - это контейнер для напитков, основанный на фрактальной геометрии, второе - это мерцающий свет, имитирующий мозговую деятельность человека. При попытке признать данные изобретения за искусственным интеллектом 22 апреля 2020 г. Управление США по патентам и товарным знакам (United States Patent and Trademark Office - USPTO) отклонило данную заявку, ссылаясь на то, что авторское право на изобретение в соответствии с патентным законодательством США может принадлежать толь- ко физическим лицам. Данное решение USPTO основано на том, что в заявке, а также сопроводительных документах имя единственного изобретателя указано как DABUS, а фамилия как «изобретение, созданное искусственным интеллектом». Стивен Талер, выступая в качестве человека, подавшего патентную заявку, пояснял, что DABUS самостоятельно задумал изобретения с использованием обученных нейронных сетей, независимо и автономно, а также не проходил специальную подготовку в соответствующей области знаний. Однако данные аргументы не были приняты и USPTO выпустило документ, известный как «Notice to File Missing Parts» (Уведомление о подаче недостающих частей)». В нем подчеркивалось, что исходя из данных, указанных в заявке, невозможно идентифицировать изобретателя по его юридическому имени. Последующие попытки Стивена Талера обжаловать данное решение, а также попытки зарегистрировать систему DABUS в качестве изобретателя в Европе и Великобритании, пока не увенчались успехом [10].

На сегодняшний день патентные законы во всем мире, в том числе и России, предоставляют права на изобретения только людям. Так, в ч. 4 Гражданского кодекса Российской Федерации указано, что изобретателем признается гражданин, творческим трудом которого создан соответствующий результат интеллектуальной деятельности [1, ст. 1347]. Тем не менее уже сейчас существуют машины, которые вносят значительный вклад в разработку новых технологий. Возможно, на данном этапе не имеет смысла целиком предоставлять права на интеллектуальную собственность компьютерам, поскольку они ни морально, ни юридически не обязаны защищать эти права. В данном контексте более целесообразным является подход, который закрепляет права на изобретение за физическим или юридическим лицом, которые изначально способствовали созданию самого программного обеспечения для компьютера с искусственным интеллектом.

\section{Ответственность AI за нарушения патентных прав}

Другим немаловажным аспектом применения технологии искусственного интеллекта 
в сфере интеллектуальной собственности является вопрос нарушения со стороны AI патентных прав.

Как и в ситуации с правами на изобретение, о которой уже упоминалось в настоящей статье, проблема ответственности за действия искусственного интеллекта стоит очень остро. В большинстве юрисдикций под патентными правами понимается предоставление автору изобретения, полезной модели или промышленного образца исключительного права, а также права авторства.

Кроме того, автору изобретения, полезной модели или промышленного образца принадлежат право на получение патента, право на вознаграждение за служебное изобретение, полезную модель или промышленный образец. Также патентные права включают право изобретателей исключать других лиц от практики использования запатентованных изобретений без соответствующих на то разрешений в течение всего срока действия патента.

Ситуации, когда права на патенты нарушаются физическими или юридическими лицами, случаются довольно часто, и меры ответственности за подобные нарушения определены действующим патентным законодательством. Однако в случае нарушения данных прав со стороны искусственного интеллекта в законе существует пробел. Ответы на данные вопросы должны способствовать развитию и совершенствованию системы патентного права.

В настоящее время законодательство большинства стран исходит из практики привлечения к ответственности человека, а не АI [5].

Однако такой подход вызывает ряд дискуссий среди пользователей и разработчиков программного обеспечения искусственного интеллекта. Это обусловлено тем, что конечные пользователи, как правило, не могут предвидеть наступление нарушения патентных прав. Вопрос привлечения людей к ответственности за непредвиденные действия AI приводит к сдерживанию развития и использования искусственного интеллекта, а значит препятствует инновационным процессам.

С целью минимизировать риск наступления неблагоприятных последствий для создателей искусственного интеллекта была предложена система страхования, схожая с систе- мой страхования автомобилей, а также создание специальных фондов для покрытия убытков, связанных с действиями AI [7, с. 458].

Другим вариантом решения проблемы ответственности является привлечение самого искусственного интеллекта. Такой подход требует внесения существенных изменений в действующее патентное законодательство, а также признания AI юридическим лицом. Однако несмотря на достаточно широкое определение понятия юридического лица, такой исход событий возможен только в долгосрочной перспективе.

\section{Вывод}

Несомненно, что будущее управления интеллектуальной собственностью во многом зависит от использования инновационных технологий, таких как искусственный интеллект. Для своего эффективного развития системы управления интеллектуальной собственностью должны максимально быстро адаптироваться к этим изменениям.

Развитие технологий искусственного интеллекта является центральным звеном в разработке более совершенных систем управления интеллектуальной собственностью.

Временной разрыв между развитием интеллектуальной собственности и вопросами совершенствования патентного законодательства приведет к созданию альтернативных стратегий работы с интеллектуальной собственностью в будущем. Несомненно, инновационные технологии требуют новых значимых механизмов защиты IP, таких как:

- разработка новых доктрин для новых технологий;

- модификация существующей патентной системы;

- изменение политики защиты прав интеллектуальной собственности.

\section{ПРИМЕЧАНИЕ}

${ }^{1}$ Работа выполнена при финансовой поддержке гранта Президента РФ № НШ-2668-2020.6 «Национально-культурные и цифровые тренды социально-экономического и политико-правового развития Российской Федерации в XXI веке». 
This work was financially supported by the Grant of the President of the Russian Federation no. HШ-2668-2020.6 "National-Cultural and Digital Trends in the Socio-Economic, Political and Legal Development of the Russian Federation in the $21^{\text {st }}$ Century."

\section{СПИСОК ЛИТЕРАТУРЫ}

1. Гражданский кодекс Российской Федерации (часть четвертая) от 18.12.2006 № 230-Ф3 (ред. от 26.07.2019, с изм. от 24.07.2020). - Доступ из справ.-правовой системы «КонсультантПлюс».

2. Купчина, Е. В. Кибербезопасность в международном коммерческом арбитраже при рассмотрении интеллектуальных споров / Е. В. Купчина // Правовая парадигма = Legal Concept. - 2020. T. 19, № 2. - C. 129-135. - DOI: https://doi.org/ 10.15688/lc.jvolsu.2020.2.19.

3. Купчина, Е. В. Рассмотрение интеллектуальных споров в США / Е. В. Купчина // Сравнительное право и проблемы частноправового регулирования в России и зарубежных странах : сб. ст. Междунар. науч.-практ. конф. - М. : Изд-во Российского ун-та дружбы народов, 2016. - С. 89-94.

4. Мир в цифровую эпоху: политика, право и экономика XXI века / А. Ю. Мамычев, Д. А. Петрова, Я. В. Гайворонская, О. И. Мирошниченко, Ю. И. Каримова [и др.]. - М. : РИОР, 2020. - 216 с.

5. Резолюция Европарламента от 16 февраля 2017 года 2015/2013(INL) P8_TA-PROV(2017)0051.Электрон. текстовые дан. - Режим доступа: https:// www.europarl.europa.eu/doceo/document/TA-8-20170051_EN.html (дата обращения: 28.08.2020). - Загл. с экрана.

6. Development of social market economy under the influence of noneconomic factors: modeling and regulation/A. O. Inshakova, E. E. Frolova, M. V. Galkina, E. P. Rusakova // International Journal of Sociology and Social Policy. - 2020. - P. 1-11.

7. Dickenson, J. Creative machines: ownership of copyright in content created by artificial intelligence applications / J. Dickenson, A. Morgan, B. Clark // European Intellectual Property Review. 2017. - Vol. 39, № 8. - P. 457-460.

8. Digital technologies as a driver of intellectual stratification of human resources: Socio-economic inequality/ E. P. Rusakova, M. N. Dudin, O. F. Shakhov, M. S. Shakhova, Yu. S. Sizova // International Journal of Recent Technology and Engineering. -2019. - Vol. 8, № 2. - P. 4436-4440.

9. Firth-Butterfield, K. White Paper: Artificial Intelligence Collides with Patent Law/K. Firth-Butterfield, Y. Chae// World Economic Forum, Center for the Fourth Industrial Revolution, April 2018. - P. 1-24.
10. Gvoth, W. DABUS Denied: Only Natural Persons can be Named as Inventors on US Patents Blog Global Business IP and Technology Blog / William Gvoth, Douglas H. Goldhush. - Electronic text data. Mode of access: https://www.lexology.com/library/ detail.aspx?g=fd5c0397-922d-481d-a9a8-4e597d90d $3 \mathrm{ca}$. - Title from screen.

11. Lambert, P. Computer Generated Works and Copyright: Selfies, Traps, Robots, AI and Machine Learning / P. Lambert // European Intellectual Property Review. - 2017. - Vol. 39, № 1. - P. 12-20.

12. Ramalho, A. Will Robots Rule the (Artistic) World? A Proposed Model for the Legal Status of Creations by Artificial Intelligence Systems / A. Ramalho // Journal of Internet Law. - 2017. - Vol. 21, № 1. P. 12-25.

13. Rusakova, E. P. Digital rights as a new object of civil rights: issues of substantive and procedural law / E. P. Rusakova, E. E. Frolova, A. I. Gorbacheva // Advances in Intelligent Systems and Computing. 2020. - Vol. 1100. - P. 665-673.

14. The model of distribution of human and machine labor at intellectual production in industry 4.0 / A. O. Inshakova, E. E. Frolova, E. P. Rusakova, S. I. Kovalev// Journal of Intellectual Capital. - 2020. Vol. 21, № 4. - P. 601-622.

\section{REFERENCES}

1. Grazhdanskiy kodeks Rossiyskoy Federatsii (chast chetvertaya) ot 18.12.2006 № 230-FZ (red. ot 26.07.2019. s izm. ot 24.07.2020) [The Civil Code of the Russian Federation (Part four) of 18.12.2006 № 230FZ (as amended on 26.07.2019, with amendments) from 24.07.2020)]. Access from Reference Legal System 'KonsultantPlyus'.

2. Kupchina E.V. Kiberbezopasnost V mezhdunarodnom kommercheskom arbitrazhe pri rassmotrenii intellektualnykh sporov [Cybersecurity in International Commercial Arbitration when Considering Intellectual Disputes]. Pravovaya paradigma [Legal Concept], 2020, vol. 19, no. 2, pp. 129-135. DOI: https://doi.org/10.15688/ lc.jvolsu.2020.2.19.

3. Kupchina E.V. Rassmotreniye intellektualnykh sporov v SShA. Sravnitelnoye pravo i problemy chastnopravovogo regulirovaniya $v$ Rossii $i$ zarubezhnykh stranakh: sb. st. Mezhdunar. nauch.prakt. konf. [Consideration of Intellectual Disputes in the United States. In the Collection: Comparative Law and Problems of Private Law Regulation in Russia and Foreign Countries. Collection of Articles of the International Scientific and Practical Conference]. Moscow, Peoples' Friendship University of Russia, 2016, pp. 89-94. 
4. Mamychev A.Yu., Petrova D.A., Gajvoronskaya Ya.V., Miroshnichenko O.I., Karimova Yu.I., et al. Mir v cifrovuyu epohu: politika, pravo i ekonomika XXI veka [The World in the Digital Age: Politics, Law, and Economics in the $21^{\text {st }}$ Century]. Moscow, LLC “Publishing Center RIOR”, 2020. 216 p.

5. Rezolyutsiya Evroparlamenta ot 16 fevralya 2017 goda 2015/2013(INL) P8_TA-PROV(2017)0051 [Resolution of the European Parliament of February 16, 2017 2015/2013 (INL) P8_TA-PROV (2017)0051]. URL: https:/www.europarl. europa.eu/doceo/document/TA8-2017-0051_EN.html (accessed 28 August 2020).

6. Inshakova A.O., Frolova E.E., Galkina M.V., Rusakova E.P. Development of social market economy under the influence of noneconomic factors: modeling and regulation. International Journal of Sociology and Social Policy, 2020, pp. 1-11.

7. Dickenson J., Morgan A., Clark B. Creative machines: ownership of copyright in content created by artificial intelligence applications. European Intellectual Property Review, 2017, vol. 39, no. 8, pp. 457-460.

8. Rusakova E.P., Dudin M.N., Shakhov O.F., Shakhova M.S., Sizova Yu.S. Digital technologies as a driver of intellectual stratification of human resources: Socio-economic inequality. International Journal of Recent Technology and Engineering, 2019, vol. 8, no. 2, pp. 4436-4440.
9. Firth-Butterfield K., Chae Y. White Paper: Artificial Intelligence Collides with Patent Law. World Economic Forum, Center for the Fourth Industrial Revolution, April 2018, pp. 1-24.

10. William Gvoth, Douglas H. Goldhush. DABUS Denied: Only Natural Persons can be Named as Inventors on US Patents Blog Global Business IP and Technology Blog. URL: https:/www.lexology.com/ library/detail.aspx?g=fd5c0397-922d-481d-a9a84e 597d90d3ca.

11. Lambert P. Computer Generated Works and Copyright: Selfies, Traps, Robots, AI and Machine Learning. European Intellectual Property Review, 2017, vol. 39, no. 1, pp. 12-20.

12. Ramalho A. Will Robots Rule the (Artistic) World? A Proposed Model for the Legal Status of Creations by Artificial Intelligence Systems. Journal of Internet Law, 2017, vol. 21, no. 1, pp. 12-25.

13. Rusakova E.P., Frolova E.E., Gorbacheva A.I. Digital rights as a new object of civil rights: issues of substantive and procedural law. Advances in Intelligent Systems and Computing, 2020, vol. 1100, pp. 665-673.

14. Inshakova A.O., Frolova E.E., Rusakova E.P., Kovalev S.I. The model of distribution of human and machine labor at intellectual production in industry 4.0. Journal of Intellectual Capital, 2020, vol. 21, no. 4, pp. 601-622.

\section{Information About the Author}

Ekaterina V. Kupchina, Senior Lecturer, Department of Civil Law and Procedure and Private International Law, Peoples' Friendship University of Russia, Miklukho-Maklaya St, 6, 117198 Moscow, Russian Federation, belousova_ev@pfur.ru, https://orcid.org/0000-0003-1318-3654

\section{Информация об авторе}

Екатерина Валентиновна Купчина, старший преподаватель кафедры гражданского права и процесса и международного частного права, Российский университет дружбы народов, ул. Миклухо-Маклая, 6, 117198 г. Москва, Российская Федерация, belousova_ev@pfur.ru, https://orcid.org/0000-0003-1318-3654 